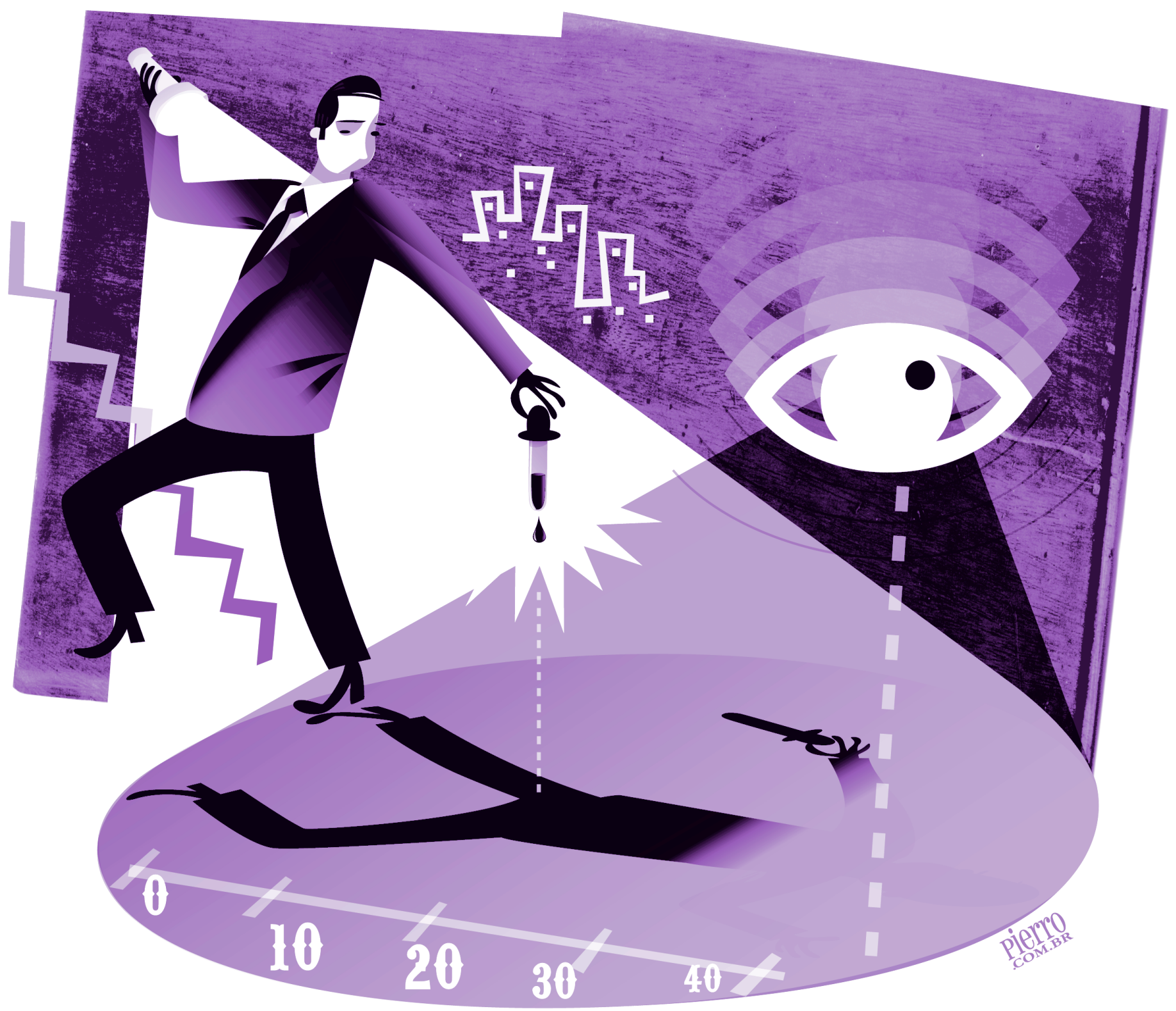




\section{Reputação organizacional: a importância de parâmetros para o seu gerenciamento}

Ana Luisa C. Almeida

- Doutora em Administração pela Universidade Federal de Minas Gerais - UFMG/ Erasmus University (Holanda)

- Mestre em Comunicação e Cultura pela Universidade Federal do Rio de Janeiro - UFRJ

- Especialista em Comunicação Integrada pela Pontifícia Universidade Católica de Minas Gerais - PUC-MG

- Graduada em Jornalismo e Relações Públicas pela PUC-MG

- Professora da Pós-graduação e graduação da PUC-MG e Fundação Dom Cabral

- Consultora em Comunicação e/ou condutora de programas de desenvolvimento para empresas

• analuisaalmeida@terra.com.br 
Resumo

O artigo aborda a importância da reputação para as organizações atuais e a necessidade de mensurá-la. Como metodologia de pesquisa aplicamos o "Quociente de Reputação", instrumento desenvolvido pelo Reputation Institute e adotado em estudos na Europa e Estados Unidos, sendo esse o primeiro estudo no Brasil. O trabalho busca contribuir com os estudos sobre as relações das organizações com seus stakeholders.

PALAVRAS-CHAVE: REPUTAÇÃO ORGANIZACIONAL • IMAGEM CORPORATIVA • COMUNICAÇÃO, MENSURAÇÃO • ESCOLA DE NEGÓCIOS

\section{Abstract}

This article discusses issues related to the corporate reputation process. This research was carried out by stakeholders of Dom Cabral Foundation, a Brazilian institution for executive management development. The research methodology adopted was the "Reputation Quotient", a method developed by the Reputation Institute and used in many European and American studies.

KEY WORDS: ORGANIZATIONAL REPUTATION - CORPORATE IMAGE - CORPORATE COMMUNICATION • MEASURING REPUTATION - BUSINESS SCHOOLS

\section{Resumen}

El artículo trata la importancia de la reputación en las organizaciones actuales y la necesidad de medirla. Como metodología de investigación aplicamos el "Cociente de Reputación”, instrumento desarrollado por el Reputation Institute y adoptado en diversos estudios en Europa y Estados Unidos, siendo éste el primer estudio en Brasil. Este trabajo pretende contribuir con los estudios sobre las relaciones de las organizaciones con sus stakeholders.

PALABRAS CLAVES: REPUTACIÓN ORGANIZACIONAL - IMAGEN CORPORATIVA - COMUNICACIÓN - MEDICIÓN - ESCUELA DE NEGOCIOS 
$\mathrm{E}$ ste artigo é parte da minha tese de doutorado $A$ influência da identidade projetada na reputação da organização que contou com a orientação do professor Reynaldo Maia Muniz, do Centro de Pós-Graduação e Pesquisas em Administração da Universidade Federal de Minas Gerais e do professor Cees B.M. van Riel, diretor do Corporate Communication Centre da Erasmus University, na Holanda. Neste estudo, pesquisamos a influência da identidade projetada sobre as percepções de stakeholders. Mais especificamente, os inter-relacionamentos entre a autoprojeção de atributos considerados pelos gestores como representativos do que seja a empresa e a percepção, pelos stakeholders, do grau de atratividade e concordância desses atributos e sua influência sobre a reputação. Como resultado final, o estudo revela aspectos que afetam direta ou indiretamente a construção da reputação, indicando elementos que devem ser considerados pelas empresas na definição de suas estratégias de relacionamentos, abordados a seguir.

\section{A importância da reputação}

Se nos perguntarmos se a reputação de uma organização é importante, provavelmente a resposta será simplesmente um "sim", mas, no entanto, a pergunta a ser feita é por que a reputação de uma empresa é importante? Pesquisas têm demonstrado que uma reputação positiva atua como um ímã, atraindo investidores, diminuindo custos do capital, trazendo novos consumidores, retendo os atuais compradores, motivando os empregados, gerando cobertura favorável da imprensa e afetando favoravelmente o conteúdo de análises financeiras (Fombrun e van Riel, 2004). A partir do momento em que se percebeu que a reputação tem impactos profundos sobre a performance corporativa, tornou-se necessário entender como se dá o processo de sua construção, como lidar com ela no dia-a-dia da organização, como sustentá-la durante os anos e como trabalhar as diversas expectativas de diferentes stakeholders ${ }^{1}$. Esse tem sido um dos grandes desafios das organizações na atualidade visto que a maioria das empresas dispõe, muitas vezes, de recursos tão similares para competir entre si, que a decisão por uma ou outra organização poderá ocorrer levando-se em conta a reputação.

1 A definição adotada nesse trabalho é a de Freeman (1984, p. 79-80) que conceitua stakeholders como "indivíduos e grupos que podem afetar ou serem afetados pelas organizações através de suas realizações". Públicos e grupos de relacionamento também serão utilizados como sinônimos de stakeholders. 
Entende-se por reputação corporativa uma representação coletiva das ações e resultados da organização, por meio da qual se demonstra sua habilidade em gerar valores para os múltiplos stakeholders (Fombrun e Rindova, 1998). Embora alguns autores utilizem os conceitos de imagem corporativa² e reputação corporativa como sinônimos, recentes estudos sobre reputação corporativa destacam duas características distintivas: reputação é construída ao longo dos anos e tem como base as ações e os comportamentos da organização (Balmer e Greyser, 2003). A reputação pode ser entendida como um crédito de confiança adquirido pela organização, estando esse crédito associado a um bom nome, familiaridade, boa vontade, credibilidade e reconhecimento. Uma reputação negativa pode significar crédito limitado ou até mesmo nenhum crédito na linha de relações com stakeholders e, mais do que uma ameaça, pode representar uma real ruptura entre empresa e ambiente (Thevissen, 2002). Uma reputação favorável aumenta a distintividade da organização, agregando-lhe vantagem competitiva, por não ser facilmente copiada (Balmer e Greyser, 2003). Por outro lado, é um recurso frágil, que não pode ser comprado e pode facilmente sofrer sérios impactos, necessitando de um gerenciamento adequado (Hall, 1992).

A literatura sobre o assunto, de acordo com Sabaté et al. (2003), revela que a reputação distingue a qualidade dos produtos (Nelson, 1970; Milgrom e Roberts, 1986); e serviços (Wilson, 1983; De Angelo, 1981; Eichenseher e Shields, 1985); assegura a garantia de contratos (Cornell e Shapiro, 1987; Sabaté et al., 2003); legitima o comportamento da firma (Fombrun, 1998; Fombrun et al., 2000; Caruana e Chircop, 2000); atua como uma força que inibe novos entrantes no setor (Kreps e Wilson, 1982; Milgrom e Roberts, 1982); e assegura mais do que a honestidade de sua conduta nas transações, o respeito aos interesses dos diversos stakeholders (Cornell e Shapiro, 1987).

\section{Por que e como mensurar a reputação?}

Gerenciar a reputação tornou-se uma ação de extrema importância para as organizações que competem no cenário atual. Percebe-se hoje a influência da reputação corporativa e seus impactos não podem mais ser ignorados (Chajet, 1997). Está havendo uma ampliação da importância da reputação empresarial como arma estratégica para gerenciamento da presença de organizações nos mercados globais. Elas são forçadas a ter uma avaliação de suas políticas corporativas, ações e interações, em qualquer país onde estejam atuando (Gardberg e Fombrun, 2002a). Com isso, as empresas sentem uma pressão maior para se diferenciar e criar políticas, ações e percepções consistentes em seus stakeholders.

2 Imagem é o conjunto de significados pelo qual um objeto é conhecido e através do qual o indivíduo se apropria para descrever, relembrar e se relacionar com o mesmo. É o resultado da interação com as crenças, idéias, sentimentos e impressões do indivíduo sobre o objeto, o qual pode ser substituído por marca ou organização. Ressalte-se, ainda, que pessoas diferentes podem ter imagens diferentes de um mesmo objeto (Dowling, 1986). 
Segundo Fombrun e Rindova (1998) o gerenciamento da reputação se justifica tanto pelo efeito sobre questões econômicas quanto por decisões estratégicas. Há evidências confirmadas sobre os ganhos financeiros obtidos como resultado de organizações com reputações fortes. Um destes estudos, de Fombrun e Shanley (1990), mostra os impactos positivos sobre a reputação quando a organização trabalha de forma eficaz sua propaganda, exerce papéis de responsabilidade social, apresenta bom desempenho e os impactos negativos, quando os lucros são voláteis e a empresa é exposta negativamente na mídia. Dados da pesquisa demonstraram que investidores reconhecem boa reputação ao decidirem pela compra de determinadas ações, consumidores pagam preços mais altos por produtos e empregados preferem trabalhar em empresas reconhecidas por sua reputação, o que, por sua vez, retorna em ganhos financeiros.

Como recurso estratégico, foi comprovado em pesquisas que uma reputação favorável significa para uma empresa ser mais atrativa do que outras aos olhos dos stakeholders, já que estes, geralmente, estabelecem seus relacionamentos com as empresas tendo como base sua reputação (Fombrun e Shanley, 1990). Hall (1992) destaca que a reputação pode ser considerada um fator de vantagem competitiva, uma vez que os competidores têm dificuldades para gerar o mesmo tipo de afeto, estima e fama criados pela reputação.

Observa-se nos estudos sobre reputação empresarial uma dualidade. De um lado, é considerada como um recurso próprio, que pode ser gerenciado pelas organizações e, por outro, como sendo as percepções de observadores, sobre as quais as organizações têm controle limitado. Evidencia-se que há dois tipos de atores envolvidos em sua formação: a organização e os observadores e, uma das conseqüências desta dualidade para as organizações é a impossibilidade de saber, de forma clara e precisa, até onde a reputação está sendo criada e gerenciada através de ações de comunicação e Marketing, políticas formais, qualidade de produtos e serviços, dentre outros aspectos, e quais as influências e interferências de outras fontes de informação e de interrelação de atores sociais (Rindova e Fombrun, 1998; Greyser, 1999; Fombrun e Shanley, 1990; Cordeiro e Samharya, 1997; Srivastava et al., 1997, Fombrun e Rindova, 1998).

Para Fombrun e Shanley (1990), a reputação é consolidada pelos stakeholders através das atividades da organização e de informações recebidas de diversas fontes. Cada grupo possui preocupações, metas e interesses específicos e lida com a organização de formas distintas. Nesse ambiente complexo de informações, observadores tentam distinguir o que define as organizações, o que persiste ao longo do tempo através das várias interações e o que é distintivo de uma organização em relação à outra num mesmo campo organizacional (Rindova e Fombrun, 1998).

Há várias formas de a organização saber como está sendo percebida pelos seus stakeholders, seja através de instâncias como fóruns públicos, notícias veiculadas na imprensa, comportamento de compra etc. (Dukerich e Carter, 2000). A mídia representa um dos principais meios de disseminar a reputação. Além do tradicional papel de disse- 
minar informações, vem também assumindo a função de vigiar a reputação das empresas (Chajet, 1997). Nos últimos anos, a imprensa, em geral, tem ampliado o espaço de cobertura de notícias sobre negócios e empresas, reconhecendo o interesse maior da sociedade por esses temas.

\section{Reputação e comunicação}

Entre as décadas de 1950 e 1970, o que se via era uma preocupação muito grande em relação à imagem que públicos externos tinham da organização e uma valorização dos elementos gráficos e design (Martineau, 1958; Caruana e Chircop, 2000). Observavase também uma grande valorização da propaganda.

O que se notou na década de 1970 e no início da de 1980, foi uma mudança expressiva na estratégia corporativa, que passou a considerar identidade e cultura organizacional em vez de ater-se somente à identificação visual (Caruana e Chircop, 2000).

A partir do final da década de 1980, o foco muda para a reputação corporativa, que leva em conta não somente a imagem atual da empresa, como também o comportamento ao longo dos últimos anos (Fombrun e Shanley, 1990). Como a organização interage com públicos distintos, que levam em consideração atributos diferentes e, portanto, têm uma visão bem diversificada da corporação, é que se faz necessário o gerenciamento da reputação.

Um gerenciamento de reputação eficiente requer uma forte capacidade de "enxergar" a habilidade de monitorar as expectativas de cada stakeholder, bem como a capacidade de antecipar eventos inesperados que podem ameaçar a reputação corporativa (Fombrun e Rindova, 1998, p. 206).

É importante, também, que a empresa, através de seu gerenciamento, consiga identificar quais os atributos são realmente importantes para cada um de seus stakeholders, podendo assim trabalhá-los de forma eficiente através de sua comunicação (Dowel et al., 1997). Somando-se às ações gerenciais, uma política de comunicação eficaz pode contribuir significativamente para criar e sustentar a reputação. Entendemos o processo de Comunicação Organizacional como um fenômeno social que pode ser visto como ações intencionais que ocorrem em contextos sociais estruturados.

Através da comunicação, a organização desperta a consciência, promove o entendimento e busca a aceitação de suas metas, idealisticamente, resultando na satisfação dos interesses de ambos: organização e ambiente (van Riel, 2000, p. 158).

A Comunicação Organizacional, a cada dia, vem ocupando mais espaço, maior escopo e intensidade, prevalecendo em quase todos os aspectos das relações da organiza- 
ção com o ambiente. Para enfrentar os desafios da contemporaneidade, as organizações necessitam pensar, planejar e administrar estrategicamente a sua comunicação. Mas para que isso realmente ocorra, a comunicação deve ser reconhecida pela alta administração como campo essencialmente estratégico para a gestão organizacional, alinhada aos objetivos e metas organizacionais. Os estudos realizados por Fombrun e van Riel (2004) demostram que as organizações melhor posicionadas em reputação são aquelas que trabalham melhor seu processo de comunicação dentro de cinco dimensões: visibilidade, transparência, distintividade, consistência e autenticidade.

\section{Figura 1 - Comunicação e reputação}

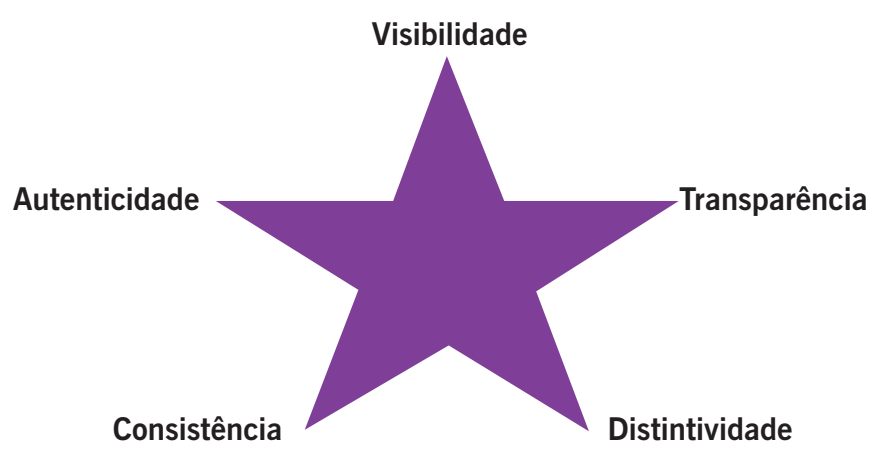

Fonte: Reputation Institute

Observa-se que, através do processo de comunicação, a organização demonstra suas habilidades, competências e conhecimentos específicos que podem lhe garantir uma posição diferenciada. E esse tem sido um grande desafio para as organizações contemporâneas. A gestão do processo de Comunicação Organizacional deve incluir uma leitura permanente das oportunidades e ameaças do ambiente e a identificação das demandas específicas de cada stakeholder considerado prioritário para a organização. Nesse sentido, mensurar a reputação contribui para seu gerenciamento, ao fornecer aos gestores elementos que orientem e embasem a tomada de decisão sobre as relações da organização com seus principais grupos de relacionamento.

\section{Metodologia de mensuração da reputação}

Grande parte dos estudos acadêmicos tem sido direcionado para a conceituação e mensuração da reputação mas, no entanto, duas grandes questões ainda não foram res- 

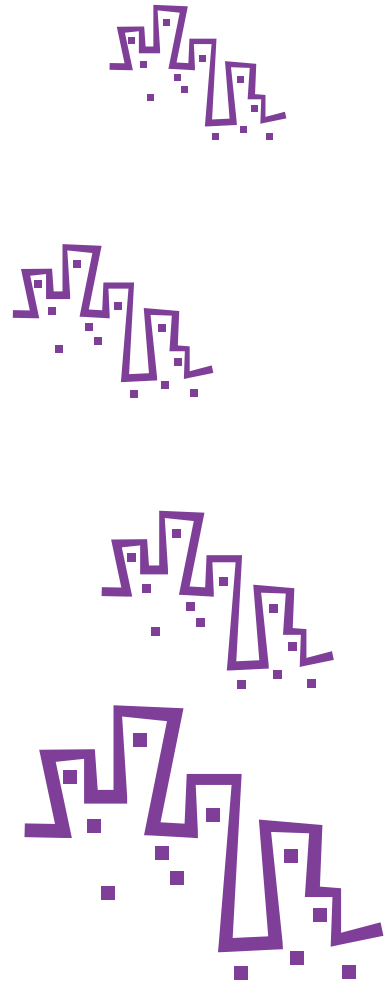

pondidas. Primeiro, ainda não se estabeleceu a definição de um único construto e, segundo, não é clara a definição dos tipos de percepções que formam a base da reputação (Berens e van Riel, 2004; Carmeli e Freund, 2002). Como conseqüência, temos várias metodologias que mensuram aspectos distintos, dependendo do tipo de associação que os indivíduos fazem sobre a organização.

Uma das metodologias considerada consistente tanto pela teoria como pelos resultados empíricos e que tem sido adotada mundialmente, foi desenvolvida por Fombrun, Gardberg e Sever, em 1999. Denominada "Quociente de Reputação", foi construída tendo como fundamentação a teoria existente sobre o tema, o desenho do instrumento e a validação do construto. Com o propósito de desenvolver uma escala que atendesse a diversos países, nos anos seguintes, foram realizadas várias pesquisas que avaliaram a "equivalência do construto", analisando em que grau a reputação organizacional tem o mesmo significado e relevância em países com culturas distintas (Fombrun, Gardberg e Sever, 2000; Gardberg e Fombrun, 2002a, p. 303). A atual metodologia foi validada após diversas pesquisas quantitativas e qualitativas realizadas pelo grupo de profissionais e acadêmicos do Reputation Institute ${ }^{3}$.

Em 2000, van Riel e Fombrun (2002), desenvolveram o projeto denominado "Projeto Global de Quociente de Reputação", realizando uma série de pesquisas em dez paí$\operatorname{ses}^{4}$, na Europa, USA, Canadá, Austrália e África do Sul com o propósito de se definir uma metodologia considerada global. As discussões sobre os resultados dessas pesquisas foram apresentadas em vários artigos publicados na edição especial da Corporate Reputation Review ${ }^{5}$, com comparações e análises sobre o processo de condução da pesquisa e as especificidades dos diversos países, a validação das dimensões propostas na metodologia, critérios e forma de processamento dos dados (van Riel e Fombrun, 2002; Gardberg e Fombrun, 2002; Groenland, 2002; Thevissen, 2002; Schultz et al., 2002; Wiedmann, 2002; Ravasi, 2002; van Riel, 2002; Macmillan et al., 2002; Gardberg e Fombrun, 2002b).

30 Reputation Institute foi criado em 1997 por Charles Fombrun (New York University) e Cees B. M. van Riel (Erasmus University) com o objetivo de gerar conhecimentos sobre reputação corporativa.

4 Algumas destas pesquisas já publicaram os primeiros resultados. Na Europa, a pesquisa está sendo conduzida na Bélgica por Frank Thevissen da Free University of Brussels, na Dinamarca por Majken Schultz da Copenhagen Business School, Kasper Nielsen e Simon Boege do Projektservice; na Alemanha por Klaus-Pieter Wiedmann, University of Hanover; na Itália por Davide Ravasi da University of Bocconi - Milan; na Holanda por Cees B. M. Van Riel da Erasmus University Rotterdam, no Reino Unido por Keith MacMillan, Kevin Money e Steve Downing da Henley Management College e nos EUA por Naomi Gardberg da Rutgers University e Charles J. Fombrun da Stern Business School of New York.

5 Edição especial da publicação Corporate Reputation Review, London, 2002, vol. 4, que trata das pesquisas do projeto "The Global Reputation Quotient (RQ)". 
Os estudos de van Riel e Fombrun, em conjunto com o grupo de pesquisadores do "Projeto Global de Quociente de Reputação" demonstram que, mesmo considerando as diferenças entre empresas de distintos setores, cultura de países, segmentos de públicos, dentre outras variáveis, há alguns atributos específicos que são definidores da reputação. Estudos como os de Bromley (2002); Schultz et al. (2001); Fombrun et al. (2000); Greyser (1999); Thevissen (2002); Gardberg e Fombrun (2002); van Riel e Fombrun (2002); Schultz, Nielsen e Boege (2002); Davies et al. (2003 e 2004); Carmeli e Freund (2002); Groenland (2002) mostram que grande parte dos trabalhos sobre análise de reputação tem se concentrado em mensuração. Por outro lado, Bromley (2002) enfatiza a necessidade de estudos qualitativos com o intuito de pesquisar por que determinados fatores são importantes na construção da reputação e como estes podem se combinar para gerar determinado resultado, positivo ou negativo para a empresa. São vários os estudiosos que atualmente têm destacado a importância da imagem e da reputação corporativa e a necessidade de se ampliar os estudos teóricos e empíricos sobre os temas. O estudo apresentado a seguir avaliou a reputação de uma organização junto a dois segmentos de públicos.

\section{A percepção dos stakeholders}

Este estudo, realizado numa Escola de Negócios (Business School), levou em consideração dois aspectos para justificar sua escolha: a ampliação de oferta e o crescimento da concorrência que tem se estabelecido nos últimos anos no setor de educação superior, no Brasil, e o entendimento de que a percepção que os stakeholders têm sobre estas instituições influencia suas escolhas. A pesquisa foi conduzida na Fundação Dom Cabral, instituição denominada como centro de desenvolvimento de executivos e empresas. A FDC é uma organização mineira, com 28 anos de atuação, considerada referência nacional no setor de educação para executivos, tendo entre seus clientes uma parcela significativa das quinhentas maiores empresas do país. Por ano, a Fundação Dom Cabral atende a mais de seiscentas empresas e cerca de nove mil executivos. Não só o número é expressivo como também representa grande parte do corpo gerencial das maiores empresas brasileiras. Trabalhar para o segmento das maiores empresas do país; formar seus executivos; realizar, em conjunto, atividades que visem à sustentação de seus negócios, traduz, na realidade, um esforço mais amplo que pode ser entendido como a concepção de um modelo empresarial para um país que busca se posicionar em um cenário de competitividade internacional. Em seu portfólio destacam-se atividades voltadas para o desenvolvimento empresarial, parcerias empresariais, desenvolvimento do gestor e programas de pós-graduação lato e stricto sensu.

Como instrumento de pesquisa adotamos o "Quociente de Reputação", que analisa seis dimensões da reputação: apelo emocional; visão e liderança; produtos e serviços; ambiente de trabalho; desempenho financeiro e responsabilidade social. Como afirmam Fombrun e Gardberg (2000, p.14) o "Quociente de Reputação" permite "avaliar 
como um grupo representativo de stakeholder percebe a organização através de vinte atributos que compõem as seis dimensões que constituem os pilares da reputação". A seguir descrevemos as seis dimensões do construto do "Quociente de Reputação".

- Apelo Emocional: percepção do quanto os indivíduos gostam, admiram e respeitam a organização.

- Produtos e Serviços: percepção da qualidade, inovação, valor e credibilidade de produtos e serviços.

- Desempenho Financeiro: percepções de resultados, perspectivas e risco.

- Visão e Liderança: percepção do processo de gestão da organização; de sua visão de futuro e capacidade de catalisar oportunidades de mercado.

- Ambiente de Trabalho: percepção do ambiente de trabalho; qualidade de seus empregados e da relação da organização com seus empregados.

- Responsabilidade Social: percepção de postura de cidadania empresarial ao lidar com comunidades, empregados e meio ambiente (Fombrun e Gardberg, 2000).

A Figura 2 a seguir sintetiza a metodologia do "Quociente de Reputação"

É importante destacar que algumas dimensões são mais importantes para determinado grupo, como por exemplo, investidores prestam mais atenção no desempenho financeiro que consumidores e estes, por sua vez, observam mais produtos e serviços.

Figura 2 - Mensuração e reputação

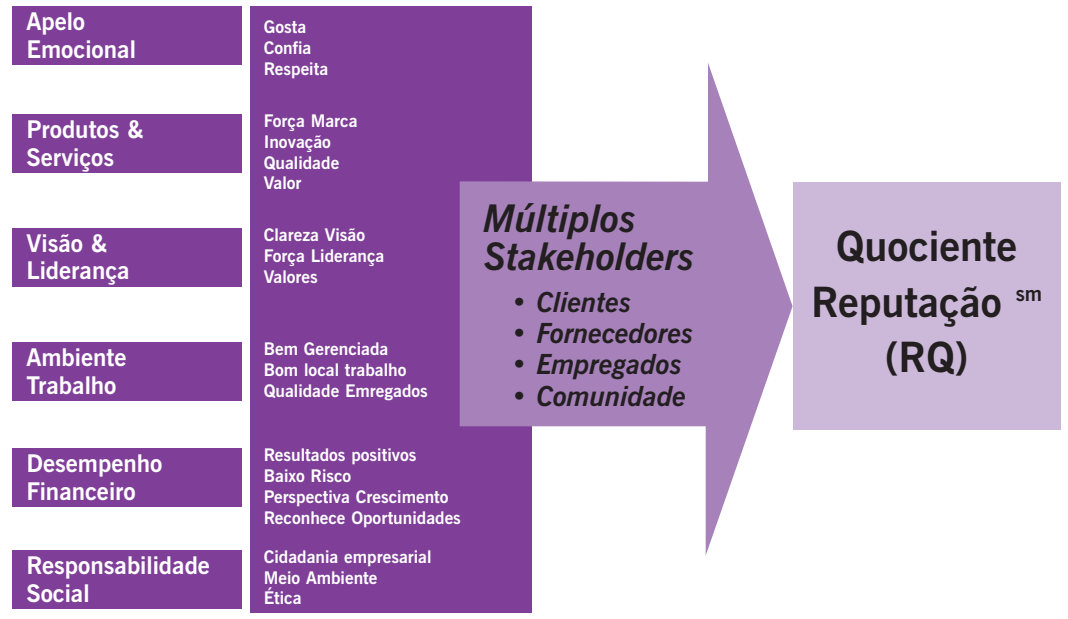


A metodologia do "Quociente de Reputação" é quantitativa. No entanto, nesse estudo, consideramos necessário incluir uma abordagem qualitativa para validarmos inicialmente as dimensões propostas no modelo e, numa segunda etapa, realizamos a pesquisa quantitativa, adotando o modelo proposto pelo Reputation Institute. A seguir, apresentamos um sumário dos resultados qualitativos e quantitativos da pesquisa realizada na Fundação Dom Cabral.

\section{A pesquisa sobre reputação na Fundação Dom Cabral}

O modelo desta pesquisa teve como propósito analisar as relações entre organização e seus stakeholders. O que a organização projeta como sendo "sua verdade" e de que forma as pessoas se apropriam ou não desta "verdade"; quem ela diz que é, quem as pessoas pensam que ela seja; o alinhamento entre o seu discurso, suas ações e as percepções de seus stakeholders. Ao conhecer estas percepções, a organização se capacita melhor para delinear uma política de relacionamento que sustente de forma mais efetiva sua reputação.

Para a seleção dos entrevistados adotou-se o seguinte critério:

1 - Empresas-clientes: compradores de produtos e serviços em 2004, representados por profissionais de recursos humanos e/ ou presidentes de empresas. Definiu-se por clientes atuais uma vez que estes possuem uma visão mais clara da atuação da FDC hoje, já que há um relacionamento estabelecido. O critério de seleção de profissionais de recursos humanos e presidentes foi adotado considerando o poder de decisão destes profissionais: compete a eles a escolha da FDC ou outra instituição concorrente para a compra de programas de desenvolvimentos de executivos para suas empresas.

2 - Executivos participantes dos programas de desenvolvimento: foram selecionados de acordo com a participação em parcerias, programas abertos, programas fechados (in company), MBAs, especialização e mestrado, uma vez, que cada um dos programas e/ ou atividades permite um tipo de relacionamento distinto com a FDC.

\section{A abordagem qualitativa}

Como instrumento de coleta de dados foram realizadas entrevistas individuais com apoio de roteiro semi-estruturado e para realização da análise dos dados foi adotada a análise de conteúdo, que teve como construção referencial de codificação as cinco dimensões que definem reputação, propostos na teoria - produtos e serviços, visão e liderança, ambiente de trabalho, responsabilidade social, desempenho financeiro. A dimensão apelo emocional é considerada uma conseqüência das outras cinco dimensões, não tendo sido objeto de análise na abordagem qualitativa. 
Os resultados da pesquisa qualitativa sobre a reputação da Fundação Dom Cabral, com empresas-clientes e executivos, indicaram que, a primeira dimensão, produtos e serviços é o que determina a reputação da FDC. Destaca-se que, agregado ao produto e serviço, está sua forma de atuar. Os depoimentos destacam a adequação do portfólio de produtos, a qualidade do corpo docente, o alinhamento com as melhores práticas nos Estados Unidos e Europa, o entendimento das necessidades dos clientes, a interface com as empresas e a forma de atendimento como principais diferenciadores de seus produtos e serviços.

Partindo do princípio de que a FDC vende para seus clientes soluções no desenvolvimento de seus executivos, considero que o mais importante são produtos e serviços (Depoimento empresa-cliente).

A FDC se distingue pelos seus produtos e serviços em dois elementos que são fundamentais: a forma como ela interage com as organizações empresariais e a partir desta visão o que ela faz e como faz. Isso na FDC é medular (Depoimento empresa-cliente).

Alguns entrevistados destacaram a forma como ela conduz o processo, a forma de fazer, "seu jeito", que pode ser traduzido no relacionamento.

É a interação. É o que ela faz de melhor. E, com essa "escuta" ela conseguiu identificar espaços para construir produtos/ portfólios bem estruturados. Não apenas na forma e abordagem, mas na seleção das instituições parceiras e/ ou professores que operam os produtos. É a forma como a FDC consegue encadear esse processo. Ela consegue cobrir as necessidades das organizações. Ela consegue cobrir desde a necessidade dos profissionais iniciantes até o desenvolvimento da cúpula. Atende a demanda dos diversos segmentos "sem excesso" (Depoimento empresa-cliente).

A segunda dimensão que obteve maior número de citação foi visão e liderança em que os entrevistados apontam para sua visão de futuro, indo além do que se espera, buscando inovação, avançando permanentemente na busca de um processo de gestão empresarial moderno, ousado e compatível com as demandas das organizações atuais.

A Fundação desfruta hoje de um conceito no meio empresarial, no meio acadêmico, melhor do que anteriormente. Esse sucesso crescente, a sua solidez e a sua constância de atuação foram plantados há muitos anos. Se a Fundação continuar com essa visão e liderança, esse trabalho vai continuar crescendo. Eu vejo que tudo o que a Fundação faz é em função dessa visão e liderança... (Depoimento executivo)

Alguns entrevistados sugeriram que sua imagem poderia ser melhor, se houvesse maior intencionalidade e abrangência no seu posicionamento externo, considerado acanhado, no sentido de ser uma instituição com características da cultura mineira, pouco afeita a uma exposição maior. Nessa mesma linha, alguns entrevistados refor- 
çaram que, ao conhecê-la melhor, a imagem também melhora. Alguns entrevistados compararam sua divulgação com instituições concorrentes, consideradas superiores nesse quesito.

\section{Resultados quantitativos}

A amostra do público externo foi dimensionada objetivando garantir níveis de certeza e precisão para os dois segmentos: empresas-clientes e executivos. O número de empresas componentes da amostra foi determinado utilizando-se um nível de confiança de $95 \%$ e um erro máximo tolerável de $5,8 \%$, o que gerou uma amostra de 174 empresas. A amostra de executivos na pesquisa foi de 551, obtida com a utilização de $95 \%$ de confiança e erro máximo de $4,1 \%$.

$\mathrm{Na}$ abordagem quantitativa, utilizou-se o instrumento de pesquisa "Quociente de Reputação", em que se avaliou as seis dimensões. Verifica-se que, na dimensão reputação, a média obtida foi de 6,29 (escala de referência: 1,0 a 7,0), revelando ser significativamente alto o grau de reputação percebido pelo público externo em relação à FDC. O resultado indica também que em todas as seis dimensões a FDC obteve índice superior a 6,0 o que demonstra que ela está bem posicionada no conjunto e também em cada dimensão isolada.

É interessante observar que, nas entrevistas qualitativas, produtos e serviços e visão e liderança foram os dois aspectos citados como determinantes de sua reputação. $\mathrm{Na}$

\section{Gráfico 1 - Dimensão da reputação}

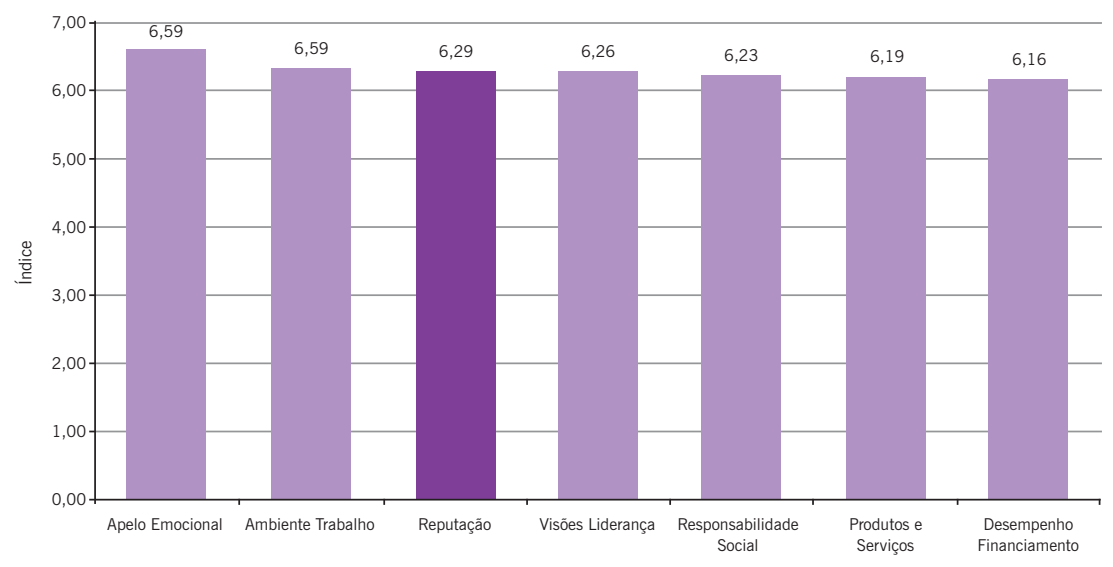


quantitativa, os aspectos são avaliados dentro de parâmetros que pontuam um conjunto de questões que compõem cada uma das dimensões. Ou seja, quando os entrevistados indicam apelo emocional e ambiente de trabalho como os itens de maior pontuação, há que se considerar que a análise é sobre "como vêem a FDC em relação a essa dimensão específica” e não o seu papel na reputação da FDC. Apelo emocional avalia em que grau as pessoas confiam, admiram e respeitam a instituição, sendo conseqüência dos outros cinco fatores.

\section{Conclusões}

Alguns críticos sociais contemporâneos, como Baudrillard, Lyotard, Deleuze têm reforçado que vivemos num mundo cada vez mais saturado por imagens e, em seus argumentos, defendem que a sociedade atual define-se pelo poder de imagens e símbolos que sustentam e apóiam os "objetos" que fazem parte de nosso dia-a-dia (Denzin, 1986).

Fazendo um recorte da sociedade para as organizações, autores nas áreas de estudos organizacionais, Comunicação e Marketing destacam o valor da imagem, afirmando que esta tem se tornado cada vez mais importante para as organizações, já que estas dependem de visibilidade e credibilidade diante de um ambiente cada vez mais confuso e hostil. E, como conseqüência da pressão que as organizações vivem, o foco da atenção nos aspectos simbólicos de suas atividades cresceu e continua em processo ascendente (Christensen e Askegaard, 2001; Cheney e Christensen, 1999). Observa-se também, nos últimos anos, o crescimento de pesquisas de diferentes perspectivas teóricas sobre dimensões simbólicas e recursos intangíveis, destacando-se dentre estes os estudos sobre reputação empresarial. Podemos afirmar assim que a reputação, como recurso intangível, tem-se tornado de alto valor por não ser possível comprá-la, imitá-la, ou adquiri-la em curto prazo.

Um dos pontos importantes observados nessa pesquisa é que há, por parte dos dois segmentos de públicos externos da FDC, um mesmo nível de entendimento do significado de seus atributos, tornando a construção de sentido mais racional e lógica. Não há dispersão de sentido e/ ou interpretações diversas e confusas sobre um mesmo atributo. Atribuímos esse entendimento à freqüência, coerência e unidade dada às informações que são transmitidas através de seu processo de comunicação, formal e informal.

Com base nos resultados da pesquisa podemos afirmar que há uma percepção de uma reputação forte e positiva. Os estudos sobre reputação ainda são pouco conhecidos no Brasil. Acreditamos que esse é um tema que muito tem a contribuir com os estudos teóricos e empíricos da Comunicação Organizacional. 


\section{Bibliografia}

ALMEIDA, A. L. C. A influência da identidade projetada na reputação da organização. Tese de Doutorado, Belo Horizonte, Centro de Pós-Graduação e Pesquisas em Administração, UFMG: 2005.

BALMER, J. M. T. e GREYSER, S. A. Revealing the Corporation: Perspectives on Identity, Image, Reputation, Corporate Branding, and Corporate-level Marketing. London: Routledge, 2003.

BERENS, G. A. J. M. e VAN RIEL, C. B. M. Corporate Associations in the Academic Literature: Three Main Streams of Thought in the Reputation Measurement Literature. Corporate Reputation Review, vol. 7, 2004, p. 161-178.

BROMLEY, D. Comparing Corporate Reputations: League Tables, Quotientes, Benchmarks or Case Studies? Corporate Reputation Review. London, vol. 5, n. 1, 2002, p. 35-50.

CARMELI, A. e FREUND, A. The Relationship between Work and Workplace Attitudes and Perceived External Prestige. Corporate Reputation Review. London, vol. 5, n. 1, 2002, p. 51-68.

CARUANA, A. e CHIRCOP, S. Measuring Corporate Reputation: A Case Example. Corporate Reputation Review. London, vol. 3, $\mathrm{n}^{\circ} 1,2000$, p. 43-57.

CHAJET, Clive. Corporate Reputation and the Bottom Line. Corporate Reputation Review. London, vol. 1, $\mathrm{n}^{\circ}$ 12, 1997, p. 19-23.

CHENEY, G. e CHRISTENSEN, L. T. Identity at Issue: Linkages between Internal and External Organizational Communication. In JABLIN, F. M. e PUTNAM, L. L. (eds.). New Handbook of Organizational Communication. Newbury Park: Sage Publications, 1999.

CHRISTENSEN, L. T e ASKEGAARD, S. Corporate Identity and Corporate Image Revisited: A Semiotic Perspective. European Journal of Marketing. Bradford, vol. 35, nº 3-4, 2001.

CORDEIRO, J. e SAMBHARYA, R. B. Do Corporate Reputations Influence Security Analysis Earnings Forecasts? Corporate Reputation Review. London, vol. 1, 1997, p. 94-98.

CORNELL, B. e SHAPIRO, A. C. Corporate stakeholders and Corporate Finance. Financial Management. vol. 16, 1987, p. 5-14.

DAVIES, G. et al. A Corporate Character Scale to Assess Employee and Customer Views of Organization Reputation. Corporate Reputation Review. London, vol. 7, n. 2, 2004.

Corporate Reputation and Competitiveness. London: Routledge, 2003.

DE ANGELO, L. Auditor Size and Auditor Quality. Journal of Accounting and Economics. vol. 3, 1981, p. 183-200.

DENZIN, N. K. Post Modern Social Theory. Sociological Theory. vol. 4, nº 2, 1986.

DOWEL, G. et al. Corporate Environment Reputation: Comparing Two Industries. Corporate Reputation Review. London, vol. 1, n 1-2, 1997.

DOWLING, G. R. Managing your Corporate Images. Industrial Marketing Management. vol. 15, 1986, p. 109-115.

EICHENSEHER, J. e SHIELDS, D. Corporate Director Liability and Monitoring Preferences. Journal of Accounting and Public Policy. vol. 4, 1985, p. 13-31. 
FOMBRUN, C. J. e RINDOVA, V. Reputation Management in Global 1000 Firms: A Benchmarking Study. Corporate Reputation Review. London, vol. 1, n³, 1998, p. 205-212.

FOMBRUN, C. J. Indices of Corporate Reputation: An Analysis of Media Rankings and Social Monitors' Ratings. Corporate Reputation Review. London, vol. 1, nº 4, 1998, p. 327-340.

FOMBRUN, C. J. e GARDBERG, N. Who's Top in Corporate Reputation? Corporate Reputation Review. London, vol. 3, n. 1, 2000, p. 13-17.

FOMBRUN, C. J.; GARDBERG, N. e SEVER, J. The Reputation Quotient: A Multi Stakeholder Measure of Corporate Reputation. The Journal of Brand Management. vol. 7, n. 4, 2000, p. 241-255.

FOMBRUN, C. J. e RINDOVA, V. P. The Road to Transparency: Reputation Management at Royal Dutch/ Shell. In SCHULTZ, Majken; HATCH, M. J. e LARSEN, M. H. The Expressive Organization: Linking Identity, Reputation and the Corporate Brand. New York: Oxford University Press, 2000.

FOMBRUN, C. J. e SHANLEY, M. What's in a Name? Reputation Building and Corporate Strategy. Academy of Management Journal. Mississippi, vol. 33, n² 2, 1990, p. 233-258.

FOMBRUN, C. J. e VAN RIEL, C. B. M. Fame \& fortune: How Successful Companies Build Winning Reputations. USA: Prentice Hall, 2004.

FREEMAN, R. E. Strategic Management: A Stakeholder Approach. Boston: Pitman, 1984.

FUNDAÇÃO DOM CABRAL. Disponível em: <http:www.fdc.org.br>.Acesso em: 12 jan. 2005.

. Portifólio. Belo Horizonte: Fundação Dom Cabral, 2004.

GARDBERG, N. A. e FOMBRUN, C. J. The Global Reputation Quotient Project: First Steps Towards a Cross-nationally Valid Measure of Corporate Reputation. Corporate Reputation Review. London, vol. 4, n. 4, 2002.

. USA: For Better or Worse: The Most Visible American Corporate Reputation. Corporate Reputation Review.

London, vol. 4, $n^{\circ}$ 4, 2002b.

GREYSER, S. A. Advancing and Enhancing Corporate Reputation. Corporate Communications. Bradford, vol. 4, n. 4, 1999, p. 177.

GROENLAND, E. Qualitative Research to Validate the RQ-Dimensions. Corporate Reputation Review. London, vol. 4, n. 4, 2002.

HALL, R. The Strategic Analysis of Intangible Resources. Strategic Management Journal. vol. 13, n 2, 1992, p. 135-144.

KREPS, D. e WILSON, R. Reputation and Imperfect Information. Journal of Economic Theory. vol. 27, 1982, p. 253-279.

KRIPPENDORFF, K. Content Analysis: An Introduction to its Methodology. London: Sage Publications, 1980.

MAcMILLAN, K.; MONEY, K. e DOWNING, S. United Kingdom: Best and Worst Corporate Reputations Nominations by the General Public. Corporate Reputation Review. London, vol. 4, n. 4, 2002.

MARTINEAU, P. Sharper Focus for the Corporate Image. Harvard Business Review, nov.-dez. 1958, p. 49-58. In BALMER, J. M. T. e GREYSER, S. A. Revealing the Corporation: Perspectives on Identity, Image, Reputation, Corporate Branding and Corporate-level Marketing. London: Routledge, 2003. 
MILGROM, P. e ROBERTS, J. Price and Advertising Signals of Product Quality. Journal of Political Economy. vol. 94, 1986, p. 796-821.

Predation, Reputation and Deterrence. Journal of Economic Theory. vol. 27, 1982, p. 280-312.

NELSON, P. Information and Consumer Behavior. Journal of Political Economy, vol. 78, 1970, p. 311-329.

RAVASI, D. Italy: Analysing Reputation in a Cross-national Setting. Corporate Reputation Review. London, vol. 4, n. 4, 2002.

RINDOVA, V. P. e FOMBRUN, C. J. The Eye of the Beholder: The Role of Corporate Reputation in Defining Organizational Identity. In WHETTEN, D. A. e GODFREY, P. C. Identity in Organizations: Building Theory through Conversations. Thousand Oaks, California: Sage Publications, 1998.

SABATÉ, J. M. de la; FUENTE e PUENTE, E. Q. The Concept and Measurement of Corporate Reputation: An Aplication to Spanish Financial Intermediares. Corporate Reputation Review. London, vol. 5, n. 4, 2003, p. 280-301.

SCHULTZ, M.; NIELSEN, K. U. e BOEGE, S. Nominations for the Most Visible Companies for the Danish RQ. Corporate Reputation Review. London, vol. 4, n. 4, 2002.

SCHULTZ, M.; MOURITSEN, J. e GABRIELSEN, G. Sticky Reputation: Analyzing a Ranking System. Corporate Reputation Review. London, vol. 4, n. 1, 2001, p. 24-41.

SRIVASTAVA, R. K. et al. The Value of Corporate Reputation: Evidence from Equity Markets. Corporate Reputation Review. London, vol. 1, 1997, p. 62-68.

THEVISSEN, F. Belgium: Corporate Reputation in the Eye of the Beholder. Corporate Reputation Review. London, vol. 4, n. 4, 2002.

VAN RIEL, C. B. M. Corporate Communication Orchestrated by a Sustainable Corporate Story. In SCHULTZ, M.; HATCH, M. J. e LARSEN, M. H. The Expressive Organization: Linking Identity, Reputation and the Corporate Brand. New York: Oxford University Press, 2000, 272 p.

. The Netherlands: Top of Mind Awareness of Corporate Brands among the Dutch Public. Corporate Reputation Review. London, vol. 4, n. 4, 2002.

. Essentials of Corporate Communication: Building and Implementing Corporate Stories Using Reputation

Management. London: Routledge, no prelo.

VAN RIEL,C. B. M. e FOMBRUN, C. J. Which Company is Most Visible in Your Country? An Introduction to the Special Issue on the Global RQ: Project Nominations. Corporate Reputation Review. London, vol. 4, n. 4, 2002.

WIEDMANN, K-P. Germany: Analyzing the German Corporate Reputation Landscape. Corporate Reputation Review. London, vol. 4, n. 4, 2002.

WILSON, R. Auditing: Perspectives from Multiperson Decision Theory. Accounting Review. vol. 58, 1983, p. 305-318. 